\title{
Tuberculosis among people living with HIV/AIDS in the German ClinSurv HIV Cohort: long-term incidence and risk factors
}

\author{
Basel Karo ${ }^{1,2^{*}}$, Walter Haas ${ }^{1}$, Christian Kollan ${ }^{1}$, Barbara Gunsenheimer-Bartmeyer ${ }^{1}$, Osamah Hamouda',
} Lena Fiebig ${ }^{1 *}$ and the German ClinSurv HIV Study Group

\begin{abstract}
Background: Tuberculosis (TB) still presents a leading cause of morbidity and mortality among people living with HIV/AIDS (PLWHA), including those on antiretroviral therapy. In this study, we aimed to determine the long-term incidence density rate (IDR) of TB and risk factors among PLWHA in relation to combination antiretroviral therapy (cART)-status.

Methods: Data of PLWHA enrolled from 2001 through 2011 in the German ClinSurv HIV Cohort were investigated using survival analysis and Cox regression.

Results: TB was diagnosed in 233/11,693 PLWHA either at enrollment ( $N=62$ ) or during follow-up $(\mathrm{N}=171)$. The TB IDR during follow-up was 0.37 cases per 100 person-years (PY) overall [95\% Cl, 0.32-0.43], and was higher among patients who never started CART and among patients originating from Sub-Saharan Africa (1.23 and 1.20 per 100PY, respectively). In two multivariable analyses, both patients (I) who never started CART and (II) those on CART shared the same risk factors for TB, namely: originating from Sub-Saharan Africa compared to Germany (I, hazard ratio (HR);

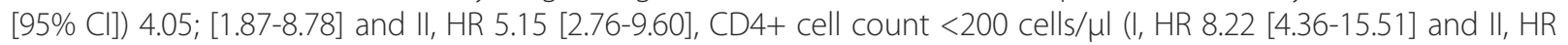
1.90 [1.14-3.15]) and viral load $>5 \log _{10}$ copies/ml (I, HR 2.51 [1.33-4.75] and II, HR 1.77 [1.11-2.82]). Gender, age or HIV-transmission risk group were not independently associated with TB.

Conclusion: In the German ClinSurv HIV cohort, patients originating from Sub-Saharan Africa, with low CD4+ cell count or high viral load at enrollment were at increased risk of TB even after CART initiation. As patients might be latently infected with Mycobacterium tuberculosis complex, early screening for latent TB infection and implementing isoniazid preventive therapy in line with available recommendations is crucial.
\end{abstract}

Keywords: Epidemiology, Incidence, HIV/AIDS, Tuberculosis, Coinfection, Antiretroviral therapy, Isoniazid preventive therapy, Industrialized country, Germany, Immigration

\section{Background}

The co-infection of tuberculosis (TB) and Human Immunodeficiency Virus (HIV) poses a major challenge to public health for both developing and industrialized countries [1]. According to WHO (2012) [2], an estimated 1.1 million new $\mathrm{TB}$ cases were reported among people living with HIV/AIDS (PLWHA). TB still presents a leading cause of morbidity and mortality among PLWHA,

\footnotetext{
* Correspondence: KaroB@rki.de; FiebigL@rki.de

1Department for Infectious Disease Epidemiology, Robert Koch Institute, Seestr. 10, 13353 Berlin, Germany

Full list of author information is available at the end of the article
}

including those on antiretroviral therapy (ART) [3]. The greatest burden of TB/HIV co-morbidity was found in Sub-Saharan Africa, where approximately 79\% of global TB/HIV cases occur [2]. Analogously, recent studies from Europe showed that Sub-Saharan African immigrants were at the highest risk of TB/HIV co-infection among the general population [4-6].

Germany is a low incidence country for TB and a low HIV-prevalence country. It has a population of 80.5 million of whom about $8 \%$ are foreign nationals and $20 \%$ are estimated to have a migration background, predominantly from Turkey, Poland and Newly Independent 
States of the Former Soviet Union [7]. The incidence of TB has continuously fallen in the general population, but the rate of decline has slowed in the last years (9.3, 7.3, 5.5 and 5.3 cases per 100,000 population in 2002, 2005, 2008 and 2011, respectively) [8]. In the same period, the number of newly diagnosed HIV cases has substantially increased (1,719 cases in 2002 vs. 2,889 cases in 2011) [9]. Individuals with foreign origin from high burden countries are considered as risk groups for both HIV and TB in Germany. Sub-Saharan Africa represents the main foreign region of origin among PLWHA. While, Turkey and the Newly Independent States of the former Soviet Union are the main foreign countries of birth among TB patients $[8,9]$. HIV prevalence in TB patients was estimated to be $4.5 \%$ in 2009 [10]. But due to the separate reporting pathways of $\mathrm{TB}$ and $\mathrm{HIV}$ in the national surveillance system [11], little is known on the TB/HIV burden and risk factors of TB among PLWHA in Germany. However, additional HIV surveillance instruments such as the German ClinSurv HIV Cohort include information on TB as an AIDS-defining disease.

The introduction of combination antiretroviral therapy (cART) in the mid-1990s has markedly reduced HIVrelated morbidity and mortality $[12,13]$. The preventive impact of ART on HIV-associated TB can be attributed to suppression of viral replication, which permits both quantitative and functional reconstitution of the host's immune system [12]. Although cART is associated with substantial declines in TB risk by 70\%-90\% among PLWHA [12], limitations of cART in reducing TB risk have been observed among some sub-populations such as elderly patients and patients with low CD4+ cell count in high-income countries [14]. A study conducted in London to investigate the effect of the initial cART on AIDS-related diseases over a 9-year period found no significant change in TB incidence before and after the introduction of cART [15]. Antiretroviral treatment might be also complicated by the immune reconstitution inflammatory syndrome (IRIS) in patients who start anti-HIV medications during TB treatment manifested by clinical deterioration in spite of increased CD4+ cell count and decreased plasma viral load [16].

Overall, little information is available on the risk profiles for TB among PLWHA living in Germany whether cART has been initiated or not.

With this study, we aimed to describe the characteristics of TB/HIV patients within the German ClinSurv HIV Cohort; to estimate the TB incidence density rate; and to identify factors associated with TB in PLWHA on cART and those who never started cART.

\section{Methods}

Data source and study population

The study was based on the German ClinSurv HIV Cohort, which is an ongoing open multicentre cohort for the clinical surveillance of HIV disease. The ClinSurv HIV cohort was established in 1999 as a collaborative cohort between specialized treatment centres for HIV/AIDS and the Robert Koch Institute (the National Public Health Institute of Germany). Irrespective of the disease stage, all HIV-infected patients are eligible to attend one of the treatment centres. After three consecutive days of clinical observation, patients will automatically be enrolled in the ClinSurv HIV Cohort. The ClinSurv dataset includes basic demographic data recorded anonymously at the first contact. Additionally, clinical, laboratory and medication history data are reported and updated at each follow-up contact. More details on the German ClinSurv HIV Cohort Study are provided by Baetzing-Feigenbaum et al. [17].

For the current analysis, data of patients enrolled in the cohort from January 2001 (introduction of the co-formulation boosted protease inhibitors Lopinavir/ Ritonavir in Germany [18]) through December 2011 were extracted from the central ClinSurv HIV database. Basic demographic characteristics including age, gender and geographic origin are recorded as time-fixed covariates at the time of enrollment. Clinical assessments (diabetes mellitus, hepatitis B and $\mathrm{C}$ ) are coded as binary covariates. Laboratory measurements (CD4+ cell counts and viral load) and medication information (TB regimens and type of ART) are coded as time-varying covariates updated in 3-month-periods when data is available.

Patients who received combination therapy involving two nucleoside reverse-transcriptase inhibitors (NRTI) plus one non-nucleoside reverse-transcriptase inhibitor (NNRTI) or protease inhibitor were defined as patients on cART. The remainder were classified as patients who never started cART during follow-up, who may however receive a different form of ART.

\section{TB/HIV case definition}

TB cases were reported including date of diagnosis; patients diagnosed with TB prior to enrollment were excluded from the analysis. TB/HIV cases were defined as any form of TB in PLWHA confirmed by positive culture of Mycobacterium tuberculosis complex. TB in PLWHA is an AIDS defining disease; therefore all TB/ HIV patients were in the $\mathrm{C}$ category according to the CDC classification system for the clinical categories of HIV infection [19].

\section{Statistical analysis}

Continuous variables were described using medians with interquartile ranges (IQR) and compared by the MannWhitney $U$-test. Categorical variables were described using numbers and percentages and compared by $\chi^{2}$ test.

For the survival analysis, the observation period was calculated as the time from enrollment to either diagnosis of 
TB, last follow-up (follow-up contact was defined by at least one visit to center every 6 months) or the end of observation period (December 2011).

The TB incidence density rate (IDR) was defined as the number of TB cases occurring per 100 patient-years (PY) of observation. The TB IDR was further stratified by gender, age group, region of origin, HIV-transmission risk group, baseline CD4+ cell count, baseline viral load, diabetes mellitus, hepatitis infections and cART-status. $P$-value was obtained by $\chi^{2}$ test for difference in TB IDRs among different groups. To compare TB IDRs after controlling for region of origin and age, the Mantel-Haenszel test was applied. Trend analyses of TB incidence were conducted using the nonparametric test for trend across ordered groups.

To compare survival probabilities between patients on cART and those who never started cART, the observation period for patients on CART was modified to start at the date when the patient first received cART and before TB diagnosis. Baseline CD4+ cell count and viral load for patients on cART were set on the date of cART initiation instead of enrollment. The endpoints of the observation remained as defined in the survival analysis. We assumed that once started on cART, patients remained on it.

The Kaplan-Meier survivor function was used to estimate TB-free survival probabilities. TB-free survival was further stratified by demographic factors, CD4+ cell count and cART-status and was compared using log-rank tests. Two multivariable Cox proportional hazards regression models, one for patients on cART and another for those never starting CART were constructed to identify factors associated with TB in relation to cART-status. Independent variables (gender, age group, region of origin, HIV-transmission risk group, baseline CD4+ cell count and viral load for patients never started cART and CD4+ cell count and viral load fixed at the time of cART initiation for the patients on cART) with $P<0.02$ in the log-rank test were included in the Cox regression models. The proportionality assumption of the final model was checked using the likelihood-ratio test and the Schoenfeld and Scaled Schoenfeld residuals. These applied tests indicated no violation of the model's proportionality. Furthermore, the goodness of fit of the final model was evaluated by the Cox-Snell residuals.

All tests were two sided with $95 \%$ confidence interval (CI); the level of significance was $P<0.05$. All analyses were performed using STATA (version12, StataCorp, LP, TX, USA) software.

\section{Ethical statement}

The ClinSurv HIV study protocol was approved by the German Federal Commissioner for Data Protection and Freedom of Information who stated that written informed consent from the patients was not required due to the anonymous nature of the data.

\section{Results}

\section{Characteristics of patients}

From January 2001 through December 2011, a total of 11,865 patients were newly enrolled in the ClinSurv HIV cohort. Of them, 172 patients were known to have TB prior to enrolment and were therefore excluded from further analyses. Of a total of 11,693 eligible HIV-positive patients, $80 \%(\mathrm{~N}=9,388)$ were men, $73 \%(\mathrm{~N}=8,362)$ originated from Germany and $12 \%(\mathrm{~N}=1,403)$ from SubSaharan Africa (Figure 1). About $80 \%$ of PLWHA received cART during the follow-up. Patients aged $>38$ years and with low baseline CD4+ cell count and high viral load were more likely to receive cART (multivariable logistic regression; $P<0.05$ ). No significant difference was found in the distribution of cART between patients from German and Sub-Saharan Africa $(P=0.79)$ (Table 1).

Of 11,693 patients, 233 were diagnosed with TB $(\mathrm{N}=62)$ at enrollment and $(\mathrm{N}=171)$ during follow-up respectively. The majority of TB/HIV co-infected patients were male (167 cases $(72 \%))$. A large proportion of the female patients originated from Sub-Saharan Africa (41 cases (64\% of 64 female patients)), while Germany was the main origin among male patients (81 cases $(51 \%$ of 159 male patients) (Table 2). At the time of enrollment, the median CD4+ cell count was significantly lower among patients who developed TB compared with those who did not (197 cells/ $\mu$ l [IQR; 67-349], 322 cells/ $\mu$ lIQR; 150-510] respectively, $P<0.001$ ). Only 130 (56\%) patients of the $233 \mathrm{~TB} / \mathrm{HIV}$ patients received cART at any point before TB diagnosis. A total of $25 \mathrm{~TB} / \mathrm{HIV}$ co-infected patients had died during the investigation period (Table 2). Additional clinical characteristics were presented in Table 2.

\section{TB incidence density rate during follow-up}

The median duration of follow-up was 40 months [IQR, 14-76]. The overall TB IDR was 0.37 cases per 100 personyears [95\% CI, 0.32-0.43] for a total observation of 45,698 person-years (Table 3).

The TB IDR was significantly higher among women compared with men (0.49 vs. 0.34 per 100PY, respectively; $P=0.04$ ) and among patients originating from Sub-Saharan Africa compared with those from Germany (1.20 vs. 0.21 per $100 \mathrm{PY}$, respectively; $P<0.001)$. However, controlling for region of origin revealed no significant difference in TB IDRs between women and men (Mantel-Haenszel test; $P=0.1$ ).

The highest TB IDR was found among patients who never started cART compared with those on cART (1.3 vs. 0.3 cases per $100 \mathrm{PY}$ respectively; $P<0.001$ ). Furthermore, significantly higher TB IDRs were found among patients with the following clinical characteristics: baseline viral 
On average 1,078 patients were enrolled in the cohort each year

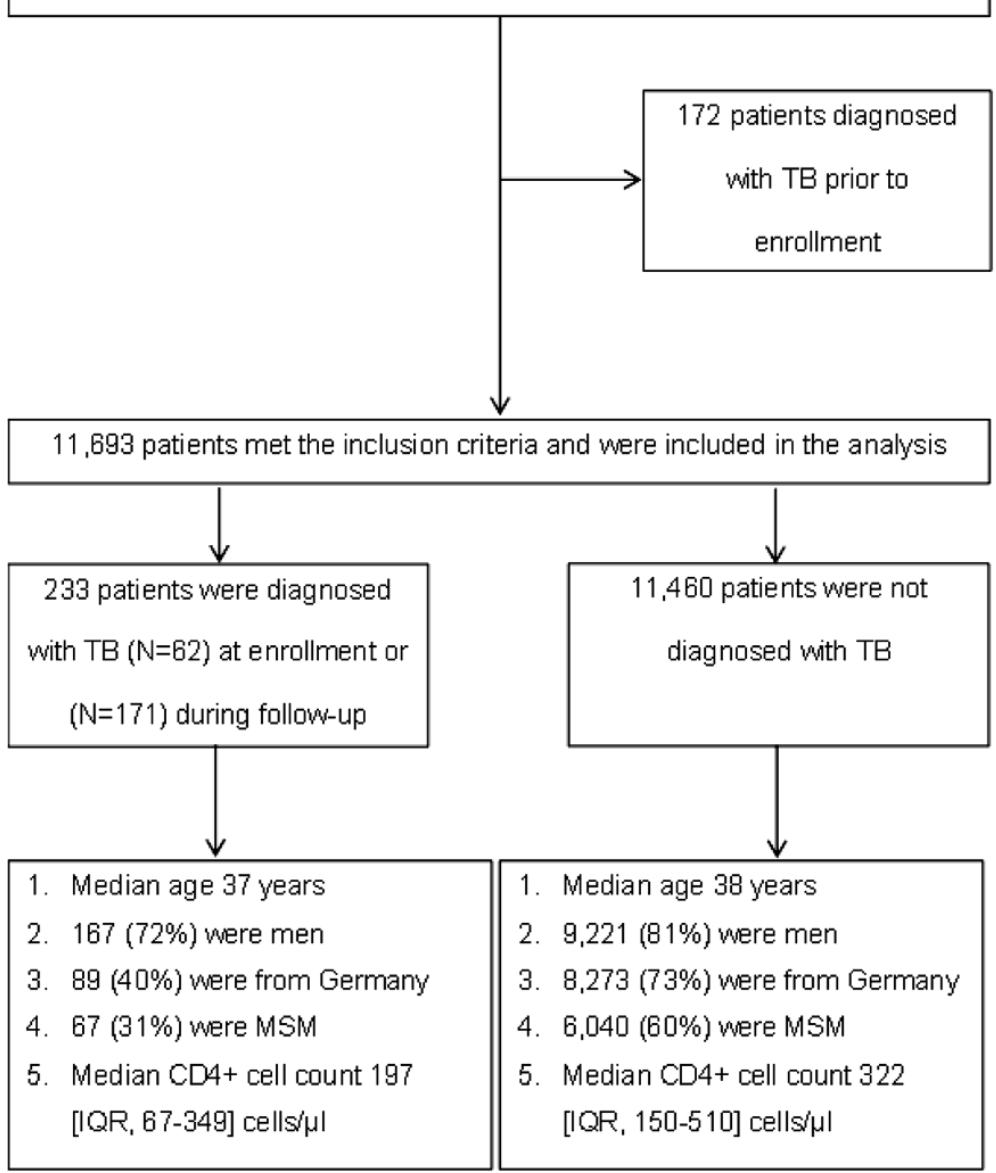

Figure 1 Overview on the patients included in the study and their characteristics at enrollment in the ClinSurv HIV Cohort, Germany 2001-2011. TB, tuberculosis; MSM, men who have sex with men; IQR, interquartile range.

load $\geq 5 \log _{10}$ copies/ml and baseline CD4+ cell count $<200$ cells $/ \mu$ l. A significantly lower TB IDR was found among men who have sex with men (MSM) compared to other HIV-transmission risk groups $(P<0.001)$.

The TB IDR was still significantly higher among patients with the aforementioned characteristics after controlling for age groups (Mantel-Haenszel test).

\section{Trend in TB incidence}

The TB IDR was highest in the first year of follow-up at 1.07 per 100PY and decreased markedly in the second year to 0.19 per $100 \mathrm{PY}(P<0.001)$ (Table 3$)$. A significant reduction in the IDR was indicated during the follow-up for both patients on CART and those who never started cART $(P<0.001$ for trend) (Figure 2). TB IDRs among patients on cART decreased further after the second year, while TB IDRs among those who never started cART tended to fluctuate and remained substantial (Figure 2).

\section{Kaplan-Meier survival probability estimates}

The TB-free survival probability for the total cohort over the 10-year follow-up was 97\% [95\% CI 96\%-98\%] (Figure 3a). The 10-year TB-free survival proportion was significantly higher among patients on cART compared with those who never started cART (98\% and 95\%, respectively; $P<0.001$ ) (Figure $3 \mathrm{~b}$ ). Among patients on cART, a significant lower TB-free survival proportion was found in patients originating from Sub-Saharan Africa compared with patients from Germany (93\% and 99\%, respectively; $P<0.001$ ) (Figure $3 \mathrm{c}$ ) and in patients with CD4+ cell count $\leq 200$ cells/ $\mu$ l compared with patients with CD4+ cell count $>200$ cells/ $\mu$ l (98\% and $99 \%$, respectively; $P<0.001$ ) (Figure $3 \mathrm{~d}$ ). The lowest TB-free survival proportion was among patients who never started cART, had a CD4+ cell count $\leq 200$ cells/ $\mu$ and originating from Sub-Saharan Africa $(60 \% ; P<0.001)$ (data not shown). 
Table 1 Demographic and clinical characteristic of people living with HIV/AIDS by cART status in the ClinSurv HIV Cohort, Germany 2001-2011

\begin{tabular}{|c|c|c|c|}
\hline Characteristic & $\begin{array}{c}\text { Non-cART } \\
\mathrm{N}=2,467(21.1 \%)\end{array}$ & $\begin{array}{c}\text { cART* }^{*} \\
\mathrm{~N}=9,226(78.9 \%)\end{array}$ & $\begin{array}{c}\text { Total } \\
\mathrm{N}=11,693(100 \%)\end{array}$ \\
\hline TB patients & $103(4.2 \%)$ & $130(1.4 \%)$ & $233(2 \%)$ \\
\hline \multicolumn{4}{|l|}{ Gender } \\
\hline Male & $1.994(80.8 \%)$ & 7,394 (80.2\%) & $9,388(80.3 \%)$ \\
\hline Female & $473(19.2 \%)$ & $1,831(19.9 \%)$ & 2,304 (19.7\%) \\
\hline \multicolumn{4}{|l|}{ Age at enrollment, years } \\
\hline Median [IQR] & $35[29-42]$ & 39 [32-46] & 38 [31-45] \\
\hline$\leq 38$ & $1,468(59.6 \%)$ & 4,338 (47.1\%) & $5,806(49.7 \%)$ \\
\hline$>38^{+}$ & 994 (40.4\%) & $4,873(52.9 \%)$ & $5,867(50.3 \%)$ \\
\hline \multicolumn{4}{|l|}{ Geographical origin } \\
\hline Germany & $1,731(72.1 \%)$ & $6,631(73.0 \%)$ & $8,362(72.8 \%)$ \\
\hline Sub-Saharan Africa & $255(10.6 \%)$ & $1,066(11.7 \%)$ & $1,321(11.5 \%)$ \\
\hline \multicolumn{4}{|l|}{ HIV-transmission risk group } \\
\hline MSM & $1,339(60.3 \%)$ & $4,768(57.8 \%)$ & 6,107 (58.4\%) \\
\hline Other & $881(39.7 \%)$ & $3,477(42.8 \%)$ & $4,358(41.6 \%)$ \\
\hline \multicolumn{4}{|c|}{ CD4+ cell count at enrollment in the cohort, cells/ $\mu \mathrm{l}$} \\
\hline Median [IQR] & 492 [349-663] & $273[118-453]$ & $320[146-508]$ \\
\hline$>200$ & 2,054 (89.1\%) & $5,467(61.9 \%)$ & $7,521(67.6 \%)$ \\
\hline$\leq 200^{\dagger}$ & $251(10.9 \%)$ & $3,362(38.1 \%)$ & $3,613(32.5 \%)$ \\
\hline \multicolumn{4}{|c|}{ Viral load at enrollment in the cohort, $\log _{10}$ copies $/ \mathrm{ml}$} \\
\hline Median [IQR] & $4.2[3.4-4.8]$ & $4.5[3.1-5.2]$ & $4.4[3.3-5.7]$ \\
\hline$<5$ & $1,769(72.9 \%)$ & $5,845(68.1 \%)$ & $7,614(70.5 \%)$ \\
\hline$\geq 5^{\dagger}$ & $446(20.1 \%)$ & $2,737(31.9 \%)$ & $3,183(29.5 \%)$ \\
\hline \multicolumn{4}{|c|}{ Calendar year of enrollment } \\
\hline 2001 & $147(5.9 \%)$ & $765(8.2 \%)$ & $912(7.7 \%)$ \\
\hline 2002 & $166(6.7 \%)$ & $832(9.0 \%)$ & $998(8.4 \%)$ \\
\hline 2003 & $178(7.2 \%)$ & $839(10.1 \%)$ & $1,017(8.7 \%)$ \\
\hline 2004 & $205(8.3 \%)$ & $928(10.1 \%)$ & $1,133(9.7 \%)$ \\
\hline 2005 & $213(8.6 \%)$ & $908(9.8 \%)$ & $1,121(9.5 \%)$ \\
\hline 2006 & $225(9.1 \%)$ & $842(9.1 \%)$ & $1,067(9.1 \%)$ \\
\hline 2007 & $251(10.2 \%)$ & $893(10.6 \%)$ & $1,144(9.8 \%)$ \\
\hline 2008 & $217(8.8 \%)$ & $883(9.5 \%)$ & $1,100(9.4 \%)$ \\
\hline 2009 & $249(10.1 \%)$ & $835(9.1 \%)$ & $1,084(9.3 \%)$ \\
\hline 2010 & $278(11.3 \%)$ & $811(8.8 \%)$ & $1,089(9.3 \%)$ \\
\hline 2011 & $338(13.7 \%)$ & $690(7.5 \%)$ & $1,028(8.8 \%)$ \\
\hline \multicolumn{4}{|c|}{ Length of follow-up (person-years) } \\
\hline Median [IQR] & $1.2[0.3-2.8]$ & $3.1[1.3-5.9]$ & $2.5[1.1-5.3]$ \\
\hline
\end{tabular}

CART, combination antiretroviral therapy; TB, tuberculosis; IQR; interquartile range; MSM, men who have sex with men; PWID, person who inject drugs; HPC, high HIV-prevalence countries.

*CART initiated during follow-up at any time prior to TB diagnosis.

${ }^{\dagger}$ Significantly associated with receiving CART (multivariable logistic regression model including all presented characteristics as independent variables; $P<0.05$ ). 
Table 2 Demographic and clinical characteristic of people living with HIV/AIDS diagnosed with TB at enrollment and during follow-up in the ClinSurv HIV Cohort, Germany 2001-2011

\begin{tabular}{|c|c|c|c|c|}
\hline \multicolumn{2}{|l|}{ Characteristics } & $\begin{array}{c}\text { Female } \\
\mathrm{N}=66(28.3 \%)\end{array}$ & $\begin{array}{c}\text { Male } \\
\mathrm{N}=167(71.7 \%)\end{array}$ & \multirow{2}{*}{ 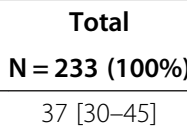 } \\
\hline Median age at enrollment ( $\mathrm{N}=232 / 1$ missing data) & Median [IQR] & $31[26-45]$ & $40[32-47]$ & \\
\hline Median age at the time of TB diagnosis, years ( $N=251 / 1$ missing data) & Median $[\mathrm{IQR}]$ & 32 [28-39] & $40[32-48]$ & $37[31-45]$ \\
\hline Region of origin ( $\mathrm{N}=223 / 10$ missing data) & N. (\%) & & & \\
\hline Germany & & $8(12.5)$ & $81(50.9)$ & $89(39.9)$ \\
\hline Sub-Saharan Africa & & $41(64.1)$ & $41(25.8)$ & $82(36.8)$ \\
\hline Other countries & & $15(23.4)$ & $37(23.3)$ & $52(23.3)$ \\
\hline HIV-transmission risk group ( $N=214 / 19$ missing data) & N. (\%) & & & \\
\hline MSM & & - & $67(43.8)$ & $67(31.3)$ \\
\hline PWID & & $3(4.9)$ & $17(11.1)$ & $20(9.4)$ \\
\hline HPC & & $54(88.5)$ & $43(28.1)$ & $97(45.3)$ \\
\hline Others $^{\dagger}$ & & $4(6.6)$ & $26(16.9)$ & $30(14.0)$ \\
\hline Median CD4+ blood cell count at enrollment ( $N=221 / 12$ missing data) & Median $[\mathrm{IQR}]$ & 197 [67-349] & $204[67-375]$ & 189 [66-348] \\
\hline $\begin{array}{l}\text { Median CD4+ blood cell count at the time of TB diagnosis } \\
(\mathrm{N}=194 / 39 \text { missing data) }\end{array}$ & Median $[\mathrm{IQR}]$ & $211[65-339]$ & 198 [47-339] & 216 [69-350] \\
\hline Median viral load at enrollment $\left(\log _{10}\right.$ copies $\left./ \mathrm{ml}\right)(\mathrm{N}=210 / 23$ missing data) & Median [IQR] & $4.8[3.6-5.4]$ & $4.7[3.4-5.5]$ & $4.9[3.6-5.3]$ \\
\hline Median viral load at the time of TB diagnosis ( $N=145 / 88$ missing data) & Median $[\mathrm{IQR}]$ & $5.0[4.3-5.6]$ & $5.2[4.5-5.7]$ & $5.9[3.9-5.5]$ \\
\hline Hepatitis $B^{*}(N=233)$ & Median [IQR] & $25(37.9)$ & $39(23.4)$ & $64(27.5)$ \\
\hline Hepatitis $C^{*}(N=233)$ & Median [IQR] & $5(7.6)$ & $18(10.8)$ & $23(9.9)$ \\
\hline Diabetes mellitus* ( $N=233$ ) & Median [IQR] & $2(3.0)$ & $5(3.0)$ & $7(3.0)$ \\
\hline $\mathrm{CART}^{\ddagger}(\mathrm{N}=233)$ & Median [IQR] & $31(50.0)$ & 99 (59.3) & $130(55.8)$ \\
\hline Died $(N=233)$ & N. (\%) & $4(10.6)$ & $18(10.8)$ & $25(10.7)$ \\
\hline
\end{tabular}

TB, tuberculosis; MSM, men who have sex with men; PWID, person who inject drugs; HPC, high HIV-prevalence countries; cART, combination antiretroviral therapy; $\mathrm{IQR}$, interquartile range.

*At any time during follow-up.

${ }^{\dagger}$ Others included heterosexual, blood transfusion and mother to child transmission.

${ }^{\ddagger} \mathrm{CART}$ initiated during follow-up at any time prior to TB diagnosis.

\section{Risk factors for tuberculosis}

In the multivariable analyses including gender, region of origin, HIV-transmission groups, CD4+ cell count and viral load as explanatory variables; risk factors of TB in patients who never started cART were: originating from Sub-Saharan Africa (Hazard ratio (HR) 4.05; 95\% CI 1.87-8.78; $P<0.001)$, CD4+ cell coun $t \leq 200$ cells $/ \mu \mathrm{l}$ (HR 8.22; 95\% CI 4.36-15.51; $P<0.001$ ) and viral load $\geq 5$ $\log _{10}$ copies $/ \mathrm{ml}$ (HR 2.51; 95\% CI 1.33-4.75; $P=0.005$ ) (Table 4).

Patients on cART shared the same risk factors of TB with the patients who never started cART, namely: originating from Sub-Saharan Africa (HR 5.15; 95\% CI 2.769.60; $P<0.001), \mathrm{CD} 4+$ cell count $\leq 200$ cells $/ \mu \mathrm{l}(\mathrm{HR}, 1.90$; $95 \%$ CI 1.14-3.15; $P=0.013$ ) and viral $\operatorname{load} \geq 5 \log _{10}$ copies/ml (HR 1.77; 95\% CI 1.11-2.82; $P=0.016$ ). Additionally, other geographic origins were significantly associated with TB risk in patients on cART (Table 4).

Although MSM was negatively associated with the diagnosis of TB in univariable analyses (data not shown), it was not associated with risk of $\mathrm{TB}$ in the multivariate analyses. Gender and age were also not independently associated with TB risk in PLWHA in both models.

\section{Discussion}

This study investigated the long-term TB incidence and risk factors among PLWHA in Germany. A specific feature of the study is that it is not restricted to patients receiving cART, but determines TB IDR and TB risk factors also for patients who never started cART. The ClinSurv HIV Cohort used in this study is characterized by a long duration of follow-up and broad enrollment criteria of PLWHA irrespective of their age, disease stage or ART-status. Therefore, this instrument allowed us to calculate long-term $\mathrm{TB}$ incidence rate and to determine associated risk factors with high precision and to update existing estimates [20]. This enhances the generalizability of the study results to other countries with low TB and HIV incidence. 
Table 3 TB incidence density rate stratified by baseline demographic and clinical characteristics in the ClinSurv HIV Cohort, Germany 2001-2011

\begin{tabular}{|c|c|c|c|c|c|}
\hline Characteristics & No. of patients & Person-years & No. with TB & TB IDR [95\% CI] & $P$ value ${ }^{*}$ \\
\hline Total patients & 11,631 & 45,698 & 170 & $0.37[0.32-0.43]$ & \\
\hline Gender & & & & & 0.04 \\
\hline Female & 2,283 & 9,153 & 45 & $0.49[0.37-0.66]$ & \\
\hline Male & 9,347 & 36,536 & 125 & $0.34[0.29-0.41]$ & \\
\hline Median age, years & & & & & 0.41 (NS) \\
\hline$\leq 38$ & 5,773 & 23,250 & 91 & $0.39[0.32-0.48]$ & \\
\hline$>38$ & 5,838 & 22,387 & 78 & $0.35[0.28-0.43]$ & \\
\hline Region of origin & & & & & $<0.001$ \\
\hline Germany & 8,344 & 33,449 & 71 & $0.21[0.17-0.27]$ & \\
\hline Sub-Sahara Africa & 1,298 & 4,871 & 59 & $1.20[0.94-1.56]$ & \\
\hline Other countries & 1,789 & 6,710 & 34 & $0.52[0.38-0.71]$ & \\
\hline HIV-transmission risk group category & & & & & $<0.001$ \\
\hline MSM & 6,093 & 24,189 & 53 & $0.22[0.17-0.28]$ & \\
\hline HPC & 1,528 & 5,910 & 69 & $1.17[0.92-1.48]$ & \\
\hline PWID & 943 & 3,534 & 14 & $0.42[0.23-0.67]$ & \\
\hline Others $^{\dagger}$ & 1,846 & 7,768 & 22 & $0.28[0.18-0.43]$ & \\
\hline CD4+ blood cell count (cells/ $\mu$ l) & & & & & $<0.001$ \\
\hline$>350$ & 5,037 & 19,702 & 44 & $0.22[0.17-0.30]$ & \\
\hline $200-350$ & 2,459 & 9,893 & 37 & $0.36[0.26-0.50]$ & \\
\hline$<200$ & 3,580 & 14,111 & 82 & $0.58[0.47-0.72]$ & \\
\hline Viral load (log 10 copies/ml) & & & & & $<0.001$ \\
\hline$<5$ & 7,583 & 30,137 & 92 & $0.31[0.25-0.37]$ & \\
\hline$\geq 5$ & 3,162 & 12,490 & 65 & $0.52[0.41-0.66]$ & \\
\hline Antiretroviral therapy & & & & & $<0.001$ \\
\hline Never started CART & 2,424 & 4,801 & 59 & $1.23[0.95-1.59]$ & \\
\hline CART during follow-up & 9,207 & 40,897 & 111 & $0.27[0.23-0.33]$ & \\
\hline Follow-up & & & & & $<0.001$ \\
\hline First year & 2,421 & 10,174 & 109 & 1.07 [0.89-1.29] & \\
\hline Second year & 1,615 & 8,248 & 16 & $0.19[0.12-0.32]$ & \\
\hline
\end{tabular}

TB, tuberculosis; IDR, incidence density rate (cases per 100 person-years); Cl, confidence interval; NS, non-significant; MSM, men who have sex with men; HPC, high HIV-prevalence countries; PWID, person who inject drugs; CART, combination antiretroviral therapy.

*Obtained by $x^{2}$ test for difference in TB IDR.

${ }^{\dagger}$ Others included heterosexual, blood transfusion and mother to child transmission.

Of 11,693 patients enrolled in the ClinSurv HIV Cohort, 233 were diagnosed with TB. Nearly 27\% $(\mathrm{N}=62)$ of TB cases were diagnosed at enrollment representing prevalent $\mathrm{TB}$ at time of clinic entry rather than true incident TB; a diagnosis of these TB episodes may have promoted HIV testing. These TB cases could potentially have been averted if individuals have been offered early HIV testing and enrolled early in a treatment center.

The TB IDR among HIV-positive patients in this study was 0.37 per 100PY; this result is consistent with findings from other studies conducted in high-income countries. A study from France showed that TB IDR was
0.4 per $100 \mathrm{PY}[6]$ and a study from the United Kingdom found that TB IDR was 0.3 per 100PY [4].

Previous studies attributed the great reduction of TB incidence, in the short term, to cART initiation [21-23]. In our study, we demonstrate a significant decrease in TB incidence density rate also among patients who never started cART during the second year. Therefore, the short-term impact of cART on TB incidence should be interpreted with caution. A higher TB rate in the first year could be due to late HIV diagnosis or closer TB monitoring in HIV-infected patients. However, the present data show that the TB incidence remained low during 10 years among patients on cART. This 


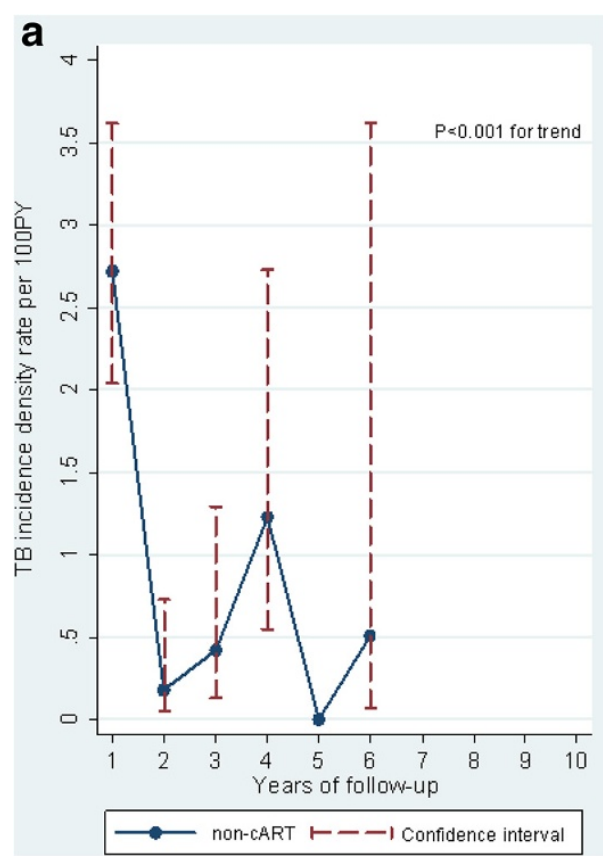

b

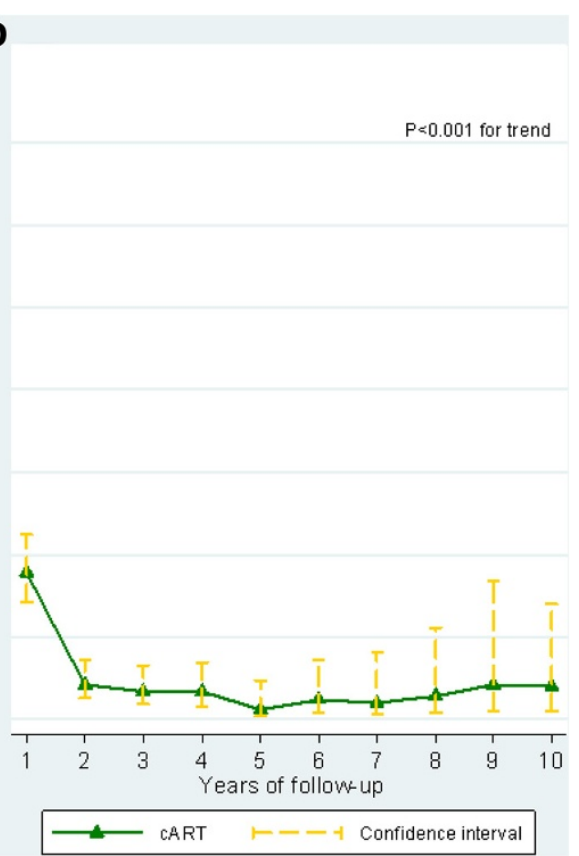

Figure 2 The trend of tuberculosis incidence density rate during follow-up, among (a) patients who never started cART $(\mathrm{N}=59)$ and (b) patients on CART $(\mathrm{N}=\mathbf{1 1 1})$ in the ClinSurv HIV Cohort, Germany 2001-2011. A low number of patients who never started CART remained under observation beyond 4 years of follow-up, where no TB cases reported in the 5th, 7th, 8th, 9th and 10th years of follow-up. $P<0.001$ for trend for TB incidence in patients who never started CART and patients on CART.

result is in agreement with findings from a recent large study (the CASCADE cohorts) [24]. Nevertheless, among patients on CART the TB rate did not further decrease in the long term. This is consistent with the immunological fact that cART has limitations in normalizing immune cell phenotype and function and fails to reduce the TB incidence to a level similar to that in an HIV-negative population $[12,25]$. It is not clear why a significant reduction in TB incidence rate among patients who never started cART occurred in the second year. One explanation could partly be a result of survival bias, as the majority of deaths in the cohort (47\%) occurred in the first year of observation. The low number of patients who never started cART as well as low TB frequency among them beyond 4 years of follow-up prevented comparison of the $\mathrm{TB}$ trend in relation to cART beyond this time point.

The data from our study confirm that originating from Sub-Saharan Africa was independently associated with increased TB risk. Similar findings were reported in another study done in France, where the relative risk of TB was 2.16 among PLWHA originating from Sub-Saharan Africa compared with patients born in France [6]. These findings can be explained in light of the high latent TB infection (LTBI) prevalence and TB incidence in their country of origin [2]. Furthermore, a wide range of socioeconomic, cultural and legal factors may also play a role in increased vulnerability to $\mathrm{TB}$ among immigrants in term of high-risk behavior and barriers to health care services [26], even though access to TB and HIV care is supposed to be free in Germany. The presumably high LTBI prevalence among PLWHA originating from Sub-Saharan Africa might expose them to a particular risk of IRIS triggered by host immune responses restored after the initiation of cART $[27,28]$.

Consistent with other findings from studies done in developing and industrialized countries [6,21-24,29,30], lower CD4+ cell count and higher viral load level at enrollment were independently associated with higher risk for TB.

Both patients on cART and those who never started cART share the same risk factors for TB. This evidence highlights the need for early screening of LTBI and offering isoniazid preventive therapy (IPT); particularly to patients originating from Sub-Sahara Africa and those with poor immune status. In the ClinSurv HIV Cohort only $0.1 \%$ of 11,693 PLWHA without TB were reported to receive isoniazid (data not shown). This might be partly related to incomplete recording of this information but could also indicate low level of IPT implementation in Germany. A similar finding was shown by a French study, which showed that IPT was rarely implemented in France in PLWHA [6].

IPT can dramatically reduce the risk of TB among PLWHA even among those receiving ART and living in 


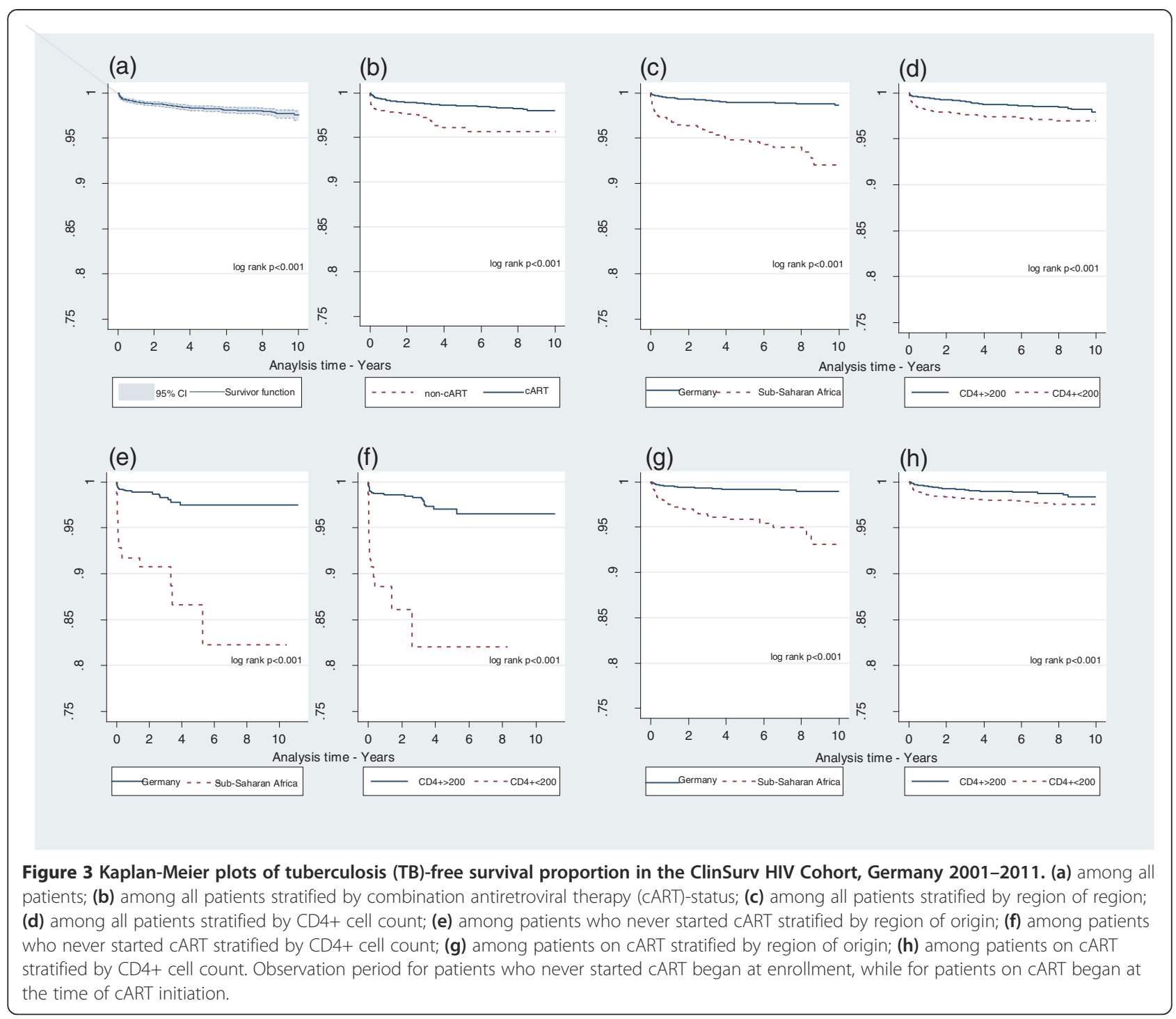

areas with low TB rates [31-33]. The new WHO guideline strongly recommends IPT to HIV patients without active TB irrespective of immune status and whether or not a person is on ART [32]. The British HIV Association Guideline identified HIV patients with increased risk for $\mathrm{TB}$ as being from sub-Saharan Africa, with CD4+ cell count $<350$ cells $/ \mu \mathrm{l}$, or duration of cART $<6$ months and recommended that these patient groups should be offered screening for LTBI and given TB chemoprophylaxis if the test result is positive [34]. Our findings support these recommendations and we therefore recommend a similar approach to be implemented in Germany.

This study has several limitations. A geographical bias related to distribution of collaborating treatment centres of the ClinSurv HIV Cohort can be noticed; cities with a relatively high notification rate of TB and HIV in Germany like Stuttgart, Frankfurt and Nuremberg [9] are left without local collaborating centres [17]. The collaborating centres are mainly specialized to treat patients with advanced disease stage, thus patients with HIV C-classification, including those with TB, might be overrepresented in the cohort. Bätzing-Feigenbaum et al. [17] also found that patients originating from high HIVprevalence countries were slightly underrepresented in the ClinSurv HIV Cohort compared to national HIV surveillance data, which could be an expression of greater difficulty in accessing specialized treatment centres. This implies that this study underestimates the actual TB/HIV burden in Germany. It is unknown whether TB diagnosis was routinely implemented to all PLWHA (active case finding) or whether it was just a part of ongoing health care (passive case finding); therefore some TB cases may not have been detected.

Discussion is also necessary on whether the TB case definition in the ClinSurv HIV Cohort (culture-positive) is strictly implemented, which might underestimate the 
Table 4 Cox proportional hazards analysis of factors associated with TB among people living with HIV/AIDS in the ClinSurv HIV Cohort, Germany 2001-2011

\begin{tabular}{|c|c|c|c|c|}
\hline \multirow[t]{2}{*}{ Predictor variable } & \multicolumn{2}{|c|}{ Patients never started cART* } & \multicolumn{2}{|c|}{ Patients on CART $^{\dagger}$} \\
\hline & HR $[95 \% \mathrm{Cl}]$ & $P$ value & $\mathrm{HR}[95 \% \mathrm{Cl}]$ & $P$ value \\
\hline \multicolumn{5}{|l|}{ Gender } \\
\hline Female & 1 & & 1 & \\
\hline Male & $0.73[0.36-1.47]$ & 0.38 (NS) & $1.49[0.84-2.62]$ & 0.17 (NS) \\
\hline \multicolumn{5}{|l|}{ Median age, years } \\
\hline$\leq 38$ & 1 & & 1 & \\
\hline$>38$ & $0.86[0.47-1.58]$ & 0.62 (NS) & 1.420 .892 .27 & 0.14 (NS) \\
\hline \multicolumn{5}{|l|}{ Region of origin } \\
\hline Germany & 1 & & 1 & \\
\hline Sub-Saharan Africa & $4.05[1.87-8.78]$ & $<0.001$ & $5.15[2.76-9.60]$ & $<0.001$ \\
\hline Other countries & $1.75[0.81-38.1]$ & 0.16 & $2.22[1.18-4.20]$ & 0.014 \\
\hline \multicolumn{5}{|l|}{ HIV-transmission risk group } \\
\hline Others $^{\dagger}$ & 1 & & 1 & \\
\hline MSM & $0.68[0.31-1.51]$ & 0.34 & $0.60[0.32-1.10]$ & 0.10 (NS) \\
\hline \multicolumn{5}{|l|}{ CD4+ cell count (cells/ul) } \\
\hline$\geq 200$ & 1 & & 1 & \\
\hline$<200$ & $8.22[4.36-15.51]$ & $<0.001$ & $1.90[1.14-3.15]$ & 0.013 \\
\hline \multicolumn{5}{|l|}{ Viral load (log 10 copies/ml) } \\
\hline$<5$ & 1 & & 1 & \\
\hline$\geq 5$ & $2.51[1.33-4.75]$ & 0.005 & $1.77[1.11-2.82]$ & 0.016 \\
\hline
\end{tabular}

$\mathrm{HR}$, hazard ratio; $\mathrm{Cl}$, confidence interval; NS, non-significant; MSM, men who have sex with men.

*50 TB cases among 1,919 subjects. ${ }^{\dagger} 78$ TB cases among 6,357 subjects.

${ }^{\dagger}$ Others included heterosexual, blood transfusion and mother to child transmission.

TB burden. Some TB cases could have also been clinically diagnosed and recorded in the ClinSurv HIV Cohort (yet was not provable case-by-case), because these TB cases are also mandatorily notified within the German TB surveillance. Due to lack of data, we were unable to include some factors known to be associated with TB in our analysis such as smoking [35], homelessness [36], alcohol abuse [37], incarceration [38] and other socioeconomic factors.

\section{Conclusion}

In conclusion, the German ClinSurv HIV Cohort represents a highly valuable data source to study the occurrence of TB/HIV in Germany. A reasonable amendment to the instrument would be the systematic collection of data on LTBI and TB diagnostics done and the completion of data on IPT.

Particularly, patients originating from Sub-Saharan African countries with high incidence of $\mathrm{TB}$, and those with low CD4+ cell count and high viral load were at increased risk of TB even after cART initiation. This suggests that actions beyond timely cART initiation should be considered; including early screening for LTBI and offering IPT in line with available recommendations.

\section{Abbreviations}

HIV: Human immunodeficiency virus; AIDS: Acquired immunodeficiency syndrome; PLWHA: People living with HIV/AIDS; CART: Combination antiretroviral therapy; TB: Tuberculosis; IRIS: Immune reconstitution inflammatory syndrome; NRTI: Nucleoside reverse transcriptase inhibitor; NNRTI: Non-nucleoside reverse transcriptase inhibitor; CDC: Centres for disease control and prevention; IQR: Interquartile rang; IDR: Incidence density rate; PY: Person-year; Cl: Confidence interval; MSM: Men who have sex with men; HPC: High HIV-prevalence countries; PWID: Person who inject drugs; HR: Hazard ratio; LTBI: latent TB infection; IPT: Isoniazid preventive therapy.

\section{Competing interests}

The authors declare that they have no competing interests.

\section{Authors' contributions}

Concept and design ( $\mathrm{LF}, \mathrm{BK}, \mathrm{WH})$, statistical analyses (BK), interpretation of the data (BK, WH, CK, BGB, OH, LF), drafting the manuscript (BK) and critical revision of the manuscript for important intellectual content $(W H, C K, B G B$, $\mathrm{OH}, \mathrm{LF}$ ). All authors read and approve the final manuscript.

\section{Authors' information}

The German ClinSurv HIV Study Group: Berlin: Robert Koch Institute: A. Kühne (Cohort manager); Vivantes Auguste-Viktoria-Hospital: K. Arastéh; Charité University Medicine: F. Bergmann, M. Warncke; Bochum: St.-Josef-Hospital/ Ruhr University: N. Brockmeyer, N. Mühlbächer; Bonn: University Hospital: J. Rockstroh, J. Wasmuth, S. Hass; Düsseldorf: University Hospital Düsseldorf: B. Jensen, L. Rollmann; Essen: University Hospital Essen: S. Esser, P. SchenkWestkamp; Hamburg: ifi-Institute for Interdisciplinary Medicine; A. Plettenberg, F. Kuhlendahl; ICH Studycenter: A. Adam, L. Weitner, K. Schewe, H. Goey, S. Fenske, T. Buhk, H.-J. Stellbrink, C. Hoffmann; University Hospital Eppendorf: J. van Lunzen, K. Wassmus; Hanover: Medical University Hanover: M. Stoll, 
S. Gerschmann, K. Hoeper; Kiel: University Schleswig-Holstein, Campus Kiel: H.A. Horst, S. Trautmann; Cologne: University Hospital Cologne: G. Fätkenheuer, D. Gillor, P. Schommers; Munich: University Hospital, Ludwig-Maximilian University: J. Bogner, B. Sonntag; Regensburg: University Hospital Regensburg: B. Salzberger; Rostock: University Hospital Rostock: C. Fritzsche

\section{Acknowledgements}

The authors are grateful to the patients who joined the ClinSurv HIV cohort. Furthermore, the authors would like to thank Barbara Hauer and Annicka Reuss for their input, Matthias an der Heiden for supporting some of the analyses, and Talei Lakeland and Jamie Berk for linguistic revision of the manuscript.

\section{Source of funding}

No external funding was received.

\section{Author details}

'Department for Infectious Disease Epidemiology, Robert Koch Institute, Seestr. 10, 13353 Berlin, Germany. ${ }^{2}$ Berlin School of Public Health, Charité Universitätsmedizin Berlin, Berlin, Germany.

Received: 15 November 2013 Accepted: 7 March 2014

Published: 19 March 2014

\section{References}

1. Horsburgh CR, Pozniak A: Epidemiology of tuberculosis in the era of HIV. AIDS 1993, 7(1):S109-S114

2. World Health Organization: Global Tuberculosis Control 2012. Geneva: World Health Organization; 2012. Available at: http://apps.who.int/iris/bitstream/ 10665/75938/1/9789241564502_eng.pdf. Accessed 17 April 2013.

3. Joint United Nations Programme on HIV/AIDS (UNAIDS): Global Report: UNAIDS Report on the Global AIDS Epidemic 2010. Geneva: UNAIDS; 2010. Available at: http://www.unaids.org/documents/20101123_globalreport_em. pdf. Accessed 25 March 2013.

4. Grant AD, Bansi L, Ainsworth J, Anderson J, Delpech V, Easterbrook P, Fisher M, Gazzard B, Gilson R, Gompels M, Hill T, Johnson M, Leen C, Orkin C, Phillips AN, Porter K, Post F, Walsh J, Sabin CA, United Kingdom Collaborative HIV Cohort Study Group: Tuberculosis among people with HIV infection in the United Kingdom: opportunities for prevention? AIDS 2009, 23(18):2507-2515.

5. Haar CH, Cobelens FG, Kalisvaart NA, Van der Have JJ, Van Gerven PJ, Van Deutekom H: HIV prevalence among tuberculosis patients in The Netherlands, 1993-2001: trends and risk factors. Int J Tuberc Lung Dis 2006, 10(7):768-774.

6. Abgrall S, Del Giudice P, Melica G, Costagliola D, Fhdh-Anrs CO: HIV-associated tuberculosis and immigration in a high-income country: incidence trends and risk factors in recent years. AIDS 2010, 24(5):763-771.

7. Das Statistische Bundesamt: Gesellschaft \& Staat: Bevölkerung. Wiesbaden; 2014. Available at: https://www.destatis.de/DE/ZahlenFakten/ GesellschaftStaat/Bevoelkerung/Bevoelkerung.html. Accessed 27. January 2014

8. Robert Koch Institute: Bericht zur Epidemiologie der Tuberkulose in Deutschland für 2010. Berlin: Robert Koch Institute; 2010. Available at: http:// www.rki.de/DE/Content/InfAZ/T/Tuberkulose/Download/TB2010.pdf? blob=publicationFile. Accessed 01 April 2013. [In German].

9. Robert Koch Institute: Infektionsepidemiologisches Jahrbuch meldepflichtiger Krankheiten für 2010. Berlin: RKl; 2011. Available at: http://edoc.rki.de/series/ infektionsepidemiologische-jahrbuecher/2010/PDF/2010.pdf. Accessed 01 April 2013. [In German].

10. Fiebig L, Kollan C, Hauer B, Gunsenheimer-Bartmeyer B, An der Heiden M, Hamouda O, Haas W: HIV-prevalence in tuberculosis patients in Germany, 2002-2009: an estimation based on HIV and tuberculosis surveillance data. PLoS One 2012, 7(11):e49111.

11. Kerwat $K$, Just $M$, Wulf $H$ : [The German protection against infection Act (infektionsschutzgesetz (IfSG))]. AINS 2009, 44(3):182-183.

12. Lawn $S D$, Bekker $L G$, Wood R: How effectively does HAART restore immune responses to Mycobacterium tuberculosis? Implications for tuberculosis control. AIDS 2005, 19(11):1113-1124.

13. Palmisano L, Vella S: A brief history of antiretroviral therapy of HIV infection: success and challenges. Ann Ist Super Sanita 2011, 47(1):44-48.
14. Collaboration HIV-CAUSAL: Impact of antiretroviral therapy on tuberculosis incidence among HIV-positive patients in high-income countries. Clin Infect Dis 2012, 54(9):1364-1372.

15. Ives NJ, Gazzard BG, Easterbrook PJ: The Changing Pattern of AIDSdefining Illnesses with the Introduction of Highly Active Antiretroviral Therapy (HAART) in a London Clinic. J Infect 2001, 42(2):134-139.

16. Murdoch DM, Venter WDF, Van Rie A, Feldman C: Immune reconstitution inflammatory syndrome (IRIS): review of common infectious manifestations and treatment options. AIDS Res Ther 2007, 4:9.

17. Bätzing-Feigenbaum J, Kollan C, Kühne A, Matysiak-Klose D, GunsenheimerBartmeyer B, Hamouda O, ClinSurv HIV Study Group: Cohort profile: the German ClinSurv HIV project-a multicentre open clinical cohort study supplementing national HIV surveillance. HIV Med 2011, 12(5):269-278.

18. Altmann M, An der Heiden M, Scheufele R, Hartmann K, Houareau C, Bartmeyer B, Hamouda O, German HIV-1 Seroconverter Cohort: The risk of AIDS-defining events is decreasing over time in the German HIV-1 Seroconverter Cohort. BMC Infect Dis 2012, 12:94.

19. Centers for Disease Control and Prevention (CDC): 1993 revised classification system for HIV infection and expanded surveillance case definition for AIDS among adolescents and adults. MMWR Recomm Rep 1992, 41:1-19.

20. Neumann G, Lichterfeld A: [HIV infection and tuberculosis-result of a survey]. Pneumologie 1991, 45(7):565-569. In German.

21. Kassa A, Teka A, Shewaamare A, Jerene D: Incidence of tuberculosis and early mortality in a large cohort of HIV infected patients receiving antiretroviral therapy in a tertiary hospital in Addis Ababa, Ethiopia. RSTMH 2012, 106(6):363-370.

22. Lawn SD, Badri M, Wood R: Tuberculosis among HIV-infected patients receiving HAART: long term incidence and risk factors in a South African cohort. AIDS 2005, 19(18):2109-2116.

23. Gupta A, Wood R, Kaplan R, Bekker LG, Lawn SD: Tuberculosis incidence rates during 8 years of follow-up of an antiretroviral treatment cohort in South Africa: comparison with rates in the community. PLoS One 2012, 7(3):e34156.

24. Lodi S, del Amo J, d'Arminio Monforte A, Abgrall S, Sabin C, Morrison C, Furrer H, Muga R, Porter K, Girardi E, CASCADE collaboration in EuroCoord: Risk of tuberculosis following HIV seroconversion in high-income countries. Thorax 2013, 68(3):207-213.

25. Lawn SD, Bekker LG, Miller RF: Immune reconstitution disease associated with mycobacterial infections in HIV-infected individuals receiving antiretroviral. Lancet Infect Dis 2005, 5(6):361-373.

26. European Centre for Disease Prevention and Control (ECDC): Migrant Health: HIV Testing and Counselling in Migrant Populations and Ethnic Minorities in EU/EEAVEFTA Member States. Stockholm: ECDC; 2011. Available at: http:// www.ecdc.europa.eu/en/publications/Publications/1108_TER_HIV_in_ migrants.pdf. Accessed 11 April 2013.

27. Lawn SD, Wood R, Wilkinson RJ: Changing concepts of "latent tuberculosis infection" in patients living with HIV infection. Clin Dev Immunol 2011, 2011: doi:10.1155/2011/98059.

28. Diedrich CR, Flynn JL: HIV-1/mycobacterium tuberculosis coinfection immunology: how does HIV-1 exacerbate tuberculosis? Infect Immun 2011, 79(4):1407-1417.

29. Girardi E, Sabin CA, d'Arminio Monforte A, Hogg B, Phillips AN, Gill MJ, Dabis F, Reiss P, Kirk O, Bernasconi E, Grabar S, Justice A, Staszewski S, Fätkenheuer G, Sterne JA, Antiretroviral Therapy Cohort Collaboration: Incidence of tuberculosis among HIV-infected patients receiving highly active antiretroviral therapy in Europe and North America. CID 2005, 41(12):1772-1782.

30. Sterling TR, Lau B, Zhang J, Freeman A, Bosch RJ, Brooks JT, Deeks SG, French A, Gange S, Gebo KA, John Gill M, Horberg MA, Jacobson LP, Kirk GD, Kitahata MM, Klein MB, Martin JN, Rodriguez B, Silverberg MJ, Willig JH, Eron JJ, Goedert JJ, Hogg RS, Justice AC, McKaig RG, Napravnik S, Thorne J, Moore RD, North American AIDS Cohort Collaboration on Research and Design (NA-ACCORD) of the International Epidemiologic Databases to Evaluate AIDS (leDEA): Risk factors for tuberculosis after highly active antiretroviral therapy initiation in the United States and Canada: implications for tuberculosis screening. J Infect Dis 2011, 204(6):893-901.

31. Golub JE, Saraceni V, Cavalcante SC, Pacheco AG, Moulton LH, King BS, Efron A, Moore RD, Chaisson RE, Durovni B: The impact of antiretroviral therapy and isoniazid preventive therapy on tuberculosis incidence 
in HIV-infected patients in Rio de Janeiro Brazil. AIDS 2007,

21(11):1441-1448.

32. World Health Organization: Guidelines for Intensified Tuberculosis Case-finding and Isoniazid Preventive Therapy for People Living with HIV in Resource-

constrained Settings. Geneva: World Health Organization; 2011. Available at: http://whalibdoc.who.int/publications/2011/9789241500708_eng.pdf. Accessed 22 April 2013.

33. Elzi L, Schlegel M, Weber R, Hirschel B, Cavassini M, Schmid P, Bernasconi E, Rickenbach M, Furrer H, Swiss HIV Cohort Study: Reducing tuberculosis incidence by tuberculin skin testing, preventive treatment, and antiretroviral therapy in an area of low tuberculosis transmission. Clin Infect Dis 2007, 44(1):94-102.

34. Pozniak AL, Coyne KM, Miller RF, Lipman MC, Freedman AR, Ormerod LP, Johnson MA, Collins S, Lucas SB, BHIVA Guidelines Subcommittee: British HIV Association guidelines for the treatment of TB/HIV coinfection 2011. HIV Med 2011, 12(9):517-524.

35. Chiang CY, Slama K, Enarson DA: Associations between tobacco and tuberculosis. Int J Tuberc Lung Dis 2007, 11(3):258-262.

36. Centers for Disease Control and Prevention (CDC): Trends in tuberculosisUnited States, 2012. MMWR Morb Mortal Wkly Rep 2013, 62(11):201-205. 22.

37. Fok A, Numata Y, Schulzer M, FitzGerald MJ: Risk factors for clustering of tuberculosis cases: a systematic review of population-based molecular epidemiology studies. Int J Tuberc Lung Dis 2008, 12(5):480-492.

38. Baussano I, Williams BG, Nunn P, Beggiato M, Fedeli U, Scano F: Tuberculosis incidence in prisons: a systematic review. PLoS Med 2010, 7(12):e1000381.

doi:10.1186/1471-2334-14-148

Cite this article as: Karo et al.: Tuberculosis among people living with HIV/AIDS in the German ClinSurv HIV Cohort: long-term incidence and risk factors. BMC Infectious Diseases 2014 14:148.

\section{Submit your next manuscript to BioMed Central and take full advantage of:}

- Convenient online submission

- Thorough peer review

- No space constraints or color figure charges

- Immediate publication on acceptance

- Inclusion in PubMed, CAS, Scopus and Google Scholar

- Research which is freely available for redistribution 